\title{
What number of you can read but still not see? A comment on a recent survey of carotid guidelines
}

\section{Introduction}

The administration of various therapeutic conditions is disputable. It is, in this way, frequently troublesome for singular experts to judge dispassionately which one of a few treatments is best for their patients. Along these lines, rules have advanced as an approach to encourage ideal patient results by abridging current information when it is mind boggling, developing, and troublesome for invested individuals to survey all alone. Lamentably, the way toward characterizing rules is defective, and the potential for this is highlighted in an as of late distributed efficient survey of $34 \mathrm{ebb}$ and flow universal rules managing the treatment of carotid ailment in patients with moderate or extreme asymptomatic (ACS) or symptomatic carotid stenosis (SCS) [1].

In a far reaching investigation of rule treatment recommendations from 23 nations, distributed in six dialects from 32 distinctive written work gatherings, Abbott et al. [1] have documented rule shortcomings prompting changeability in treatment suggestions. For example, 100\% of material rules supported carotid endarterectomy (CEA) for normal CEAchance SCS, and 96\% embraced CEA for normal CEA-chance ACS. Be that as it may, there was a more eminent variety in carotid supply route stenting (CAS) proposals. CAS was supported (recomretouched that it ought to or might be given) for normal CEA-hazard ACS by $63 \%$ of pertinent rules, while $30 \%$ unequivocally restricted it. CAS was embraced for normal CEA-hazard SCS by around half of material rules, while approximately $25 \%$ expressly restricted it. Supports of CAS for ACS and SCS were normal, notwithstanding the absence of randomized trial information demonstrating that CAS is at any rate in the same class as CEA or medicinal treatment alone for stroke anticipation. Randomized trial and registry evidence of the threats of CAS for normal CEA-chance patients [2,3] was under spoken to in a considerable lot of these rules.

CAS was additionally dynamically supported for patients considered at highchance for-CEA on account of vascular life structures, medicinal comorbidities, or vague reasons. Such supports were found in $49 \%$ of pertinent rules as for ACS and $84 \%$ of rules as for SCS. This is in spite of the nonattendance of any randomized information demonstrating that any strategy enhances results over restorative treatment alone, and the restricted future of numerous such patients [1].

There was additionally an outstanding variety in the consideration of medical treatment proposals. Any proposals in regards to general therapeutic treatment were incorporated into just $68 \%$ of rules with respect to ACS, and in $91 \%$ in regards to SCS. Particular proposals in regards to peri-CEA or peri-CAS therapeutic treatment were found in just half and $32 \%$ of material rules, separately, for ACS. Just $48 \%$ of rules contained such proposals with respect to SCS. Besides, when restorative treatment suggestions were incorporated, they were normally fragmented and regularly isolated from master cedural proposals and precluded from rundowns.

Maybe of more significance were the shortcomings in current worldwide rules revealed by what they have in like manner. In every one of the 34 rules recognized in the survey by Abbott et al, every procedural support were construct straightforwardly or in a roundabout way in light of old correlations of carotid endarterectomy and what is presently old restorative treatment
Frank J Veith *

Division of Vascular Surgery, New York University Langone Medical Center, New York, USA

*Author for correspondence:

fjvmd@msn.com

Submitted: 13 July 2017

Accepted: 14 July 2017

Published online: 24 July 2017 
in patients who were randomized lyears ago $[4,5]$. Likewise, when incorporated into the rules, the 30-day danger of stroke or passing said to give a general patient advantage from CEA (more often than not 3\% for ACS and 6\% for SCS) was gotten from a similar old, and now out of date, randomized trials.

The stroke counteractive action viability of medicinal treatment alone (reassuring a sound way of life and suitable utilization of medication) has enhanced by no less than $80 \%$ since the randomized trials of CEA versus restorative treatment began [6-9]. The perception of wager ter results now in patients with ACS without procedural mediation and its suggestions for patients with SCS (counting the requirement for better patient determination and more tightly periprocedural stroke and passing rate principles) have barely affected on rule proposals.

Abbott et al. highlighted the over-dependence on, and confinements of, randomized trial information in current guidelines [1]. Although randomized trials can be valuable if very much planned and deciphered without inclination, they are not the Holy Grail of proof based prescription. Randomized trials can wind up plainly out of date, might be misjudged, are not for the most part the most ideal approach to assess outcomes in routine practice, and are not suitable for noting all therapeutic questions [4,5]. Current rules frequently underutilize and underestimate quality, freely approved, non-randomized observational information. Such information, for instance, have demonstrated enhanced patient results with restorative treatment alone [6-9] and with CEA, [10-12] and a determination of significantly higher dangers from CAS $[2,3]$.

Abbott et al. likewise discovered major authoritative exclusions and different issues crosswise over guidelines [1] For example, of appropriate rules for ACS and SCS, just $7 \%$ and $12 \%$, separately, totally characterized carotid stenosis as per stenosis degree, the strategy for deciding the stenosis, and the planning and domain of any past stroke or TIA. Such definitions ought to take after specifically from the important randomized trials. The absence of target populace definition in rules supports procedural over usage by not constraining supports to quiet sub-bunches who plainly accomplished a factually noteworthy advantage in those trials.

Furthermore, rules were frequently open just by means of expert affiliations instead of well-known web search tools, were not independent, and included irregularities and ambiguities. They additionally regularly confounded procedural recommendations for ACS and SCS, neglected to incorporate a completely characterized star cedural standard that would infer a general patient advantage, and neglected to clarify constraints of rule suggestions. Besides, the wording used to abridge treatment proposals and the proof utilized as a part of making them were not institutionalized crosswise over rules, making understanding and correlations troublesome.

It is, consequently, clear that present universal carotid administration rules have genuine defects that may prompt problematic administration of patients in routine practice. Standardization of suggestions would take after from objective and exact translation of the confirmation base. The various imperfections in contemporary rules must be clarified by the contrasting and not as much as target perspectives of the person doctors and supporting gatherings keeping in touch with them or potentially blunders that their creators make $[4,5,13]$. Future rules ought to recognize that new confirmation, including further trials, might be useful in enhancing results for patients with carotid stenosis, and that such trials must incorporate better hazard stratification models and present day medicinal and procedural mediations. Be that as it may, such trials will take numerous years to finish and may not address the issues talked about in Abbott et al. review [1].

The inquiry that remaining parts is, would this be able to circumstance be made strides? The treatment of any condition will shift with various nations and zones of the world relying upon neighborhood propensities and assets. In this manner, a "one size fits all" rule approach is most likely not proper. In any case, future rules ought to recognize the imperfections in the randomized trials that frame their basis [4]. They ought to likewise incorporate other confirmation, for example, inclination coordinated trials (ideally multifocus), reviewed registries, and multi-registry analyses [3]. The criteria on which rules ought to be constructing could be concurred in light of globally and could incorporate direction of the worthiness of studies. For instance, they could incorporate direction on such issues as: i) are the essential result end-focuses and randomization proper to the inquiry, ii) is the examination adequately fuelled, iii) are the assessments contemporary; and iv) has the methodology been contrasted and other accessible medicines? After such understandings have been achieved, it would be critical that fair-minded national associations, which are not society based, could be made a request to regulate rule advancement and composing, and propose treatment choices for that nation. Such associations as of now exist in a few nations. Some may state that this approach would be awkward and not perfect, but rather than nor is the present framework.

The key lesson gained from Abbott et al. survey is that future rules ought to be composed in a way that takes out genuine blunder, irregularity, predisposition, and specialist self-intrigue, all of which can diminish rule esteem. Just along these lines will the enthusiasm of patients be better off. All things considered, encouraging enhanced patient results in routine 
practice ought to be the fundamental motivation behind rules.

\section{References}

1. Abbott AL, Paraskevas KI, Kakkos SK, et al. Systematic review of guidelines for the management of asymptomatic and symptomatic carotid stenosis. Stroke. 46(11): 3288e301 (2015).

2. Abbott AL, Adelman MA, Alexandrov AV, et al. Why calls for more routine carotid stenting are currently inappropriate: an international, multispecialty, expert review and position statement. Stroke. 44: 1186e90 (2013).

3. Paraskevas KI, Kalmykov EL, Naylor AR. Stroke/death rates following carotid artery stenting and carotid endarterectomy in contemporary administrative dataset registries: a systematic review. Eur. J. Vasc. Endovasc. Surg. 51: 3e12 (2016).

4. Veith FJ. How can good randomized controlled trials in leading journals be so misinterpreted? J. Vasc. Surg. 57: 3Se7S (2013).

5. Abbott AL. Bias in the use of randomized trials for carotid stenosis management. Gefasschirurgie. 20: $252 \mathrm{e} 7$ (2015).

6. Abbott AL. Medical (nonsurgical) intervention alone is now best for prevention of stroke associated with asymptomatic severe carotid stenosis: results of a systematic review and analysis. Stroke. 40: e573e83 (2009).

7. Naylor AR. Time to rethink management strategies in asymptomatic carotid artery disease. Nat. Rev. Cardiol. 9: 116e24 (2011).

8. Abbott AL. Stroke rates associated with asymptomatic carotid stenosis and medical treatment alone continue to fall: results of an updated meta-analysis (late breaking science abstract presented at the AHA international stroke conference, 2013). Stroke. 8: 26 (2013).

9. Hadar N, Raman G, Moorthy D, et al. Asymptomatic carotid artery stenosis treated with medical therapy alone: temporal trends and im-plications for risk assessment and the design of future studies. Cerebrovasc. Dis. 38: 163e73 (2014).

10. Silver FL, Mackey A, Clark WM, et al. Safety of stenting and endarterectomy by symptomatic status in the carotid revascularization endarterectomy versus stenting trial (CREST). Stroke. 42: 675e80 (2011).

11. Munster AB, Franchini AJ, Qureshi MI, Thapar A, Davies AH. Temporal trends in safety of carotid endarterectomy in asymp-tomatic patients: systematic review. Neurology. 85: 365e72 (2015).

12. Kumamaru H, Jalbert JJ, Nguyen LL, et al. Surgeon case volume and 30-day mortality after carotid endarterectomy among contemporary medicare beneficiaries: before and after national coverage deter-mination for carotid artery stenting. Stroke. 46: 1288e94 (2015).

13. Paraskevas KI, Veith FJ, Riles TS, Moore WS. Is carotid artery stenting a fair alternative to carotid endarterectomy for symptomatic carotid artery stenosis? Eur. J. Vasc. Endovasc. Surg. 41: 717e9 (2011). 